\title{
DIFFERENTIAL EMERGENCE OF CELLULAR MECHANISMS MEDIATING HABITUATION AND SENSITIZATION IN THE DEVELOPING APLYSIA NERVOUS SYSTEM ${ }^{1}$
}

\author{
STEPHEN G. RAYPORT ${ }^{2}$ AND JOSEPH S. CAMARDO \\ Center for Neurobiology and Behavior, Department of Psychiatry, Columbia University and New York State Psychiatric \\ Institute, New York, New York 10032
}

Received October 27, 1983; Revised March 30, 1984; Accepted April 4, 1984

\begin{abstract}
The development of the cellular substrates underlying habituation and sensitization, two simple forms of learning, was examined at a polysynaptic sensory-to-motor connection in the neural circuit mediating defensive mucus release in the marine mollusc, Aplysia californica. Animals were studied throughout juvenile life, stages 9 (40 days of development) to 12 (95 days), and into adulthood, stage 13 (120 days), starting just after metamorphosis when mucus release first becomes evident. Homosynaptic depression, which mediates habituation, was already present in its adult form in stage 9 . Heterosynaptic facilitation, which mediates sensitization, appeared in stage 10 and reached maturity during stages 11 and 12 . Thus, the development of synaptic plasticity in this curcuit occurs in discrete phases in which the gradual emergence of heterosynaptic facilitation occurs only after homosynaptic depression is well established.
\end{abstract}

The capacity to learn reflects an animal's ability to modify its behavior as a result of experience. Such changes in behavior are mediated at least in part by synaptic plasticity, that is, changes in the effectiveness of synaptic connections (Konorski, 1948; Hebb, 1949; Kandel, 1977). Little is known about how synapses acquire this capacity to undergo modulation or about the implications of developing synaptic plasticity for behavior. Is plasticity present when synapses first form, or does it develop later? If later, do all forms of synaptic plasticity at a given synapse arise simultaneously, or do they appear in a certain sequence? When plasticity develops, does it appear all at once or gradually? How does emerging plasticity affect the animal's behavior?

To explore the emergence of synaptic substrates of learning, we examined the development of synaptic mechanisms mediating habituation and sensitization in Aplysia. Habituation is the waning of a response to the slowly repeated presentation of a mild stimulus. Sensitization is the enhancement of the response to a given stimulus as a result of the presentation of a second

\footnotetext{
${ }^{1}$ This work was supported by a grant from the Klingenstein Foundation and by National Institutes of Health Grant GM-23540 to E. R. Kandel, M.D.-Ph.D. Program Fellowship Grant GM-07367 to S. G. R., and National Institute of Mental Health Training Grant MH 15175 to J. S. C. We thank Tom Capo and Susan Perritt for mariculture of juvenile Aplysia which is supported by National Institutes of Health, Animal Research Division Grant RR-01215-01. We are indebted to Drs. E. R. Kandel, V. Castellucci, F. Katz, and S. Schacher for their critical comments. Our sincere thanks to the late Dr. E. Diacumakos for her inspiration and contribution to the development of ultramicrotechniques.

${ }^{2}$ To whom correspondence should be addressed.
}

stronger and usually noxious stimulus. Habituation and sensitization occur in two well studied defensive behaviors mediated by the Aplysia abdominal ganglion-namely, gill withdrawal (Kupferman et al., 1970) and inking (Carew and Kandel, 1977) - and are likely to occur in a third defensive behavior, mucus release from the body wall (Rayport et al., 1983).

In the neural circuit for each of the three behaviors, a small number of effector cells receive input from a shared pool of sensory neurons in the RE and LE cell clusters (Castellucci et al., 1970; Kupfermann et al., 1974; Byrne, 1980, Rayport, 1981). The monosynaptic sensory-to-motor neuron EPSP in each circuit, as well as the polysynaptic EPSP evoked by nerve stimulation, shows homosynaptic depression, the cellular correlate of habituation, and heterosynaptic facilitation, the correlate of sensitization (Kandel, 1977). As the sensory cells are extremely small in early juvenile animals, we studied the polysynaptic EPSP evoked by nerve stimulation in order to follow the development of plastic capabilities. In the gill withdrawal circuit the plastic changes underlying habituation and sensitization have been traced to the presynaptic terminals of the sensory cells (Castellucci and Kandel, 1974, 1976), and the subcellular mechanisms are fairly well understood (Klein et al., 1980; Kandel and Schwartz, 1982). Since the RE and LE sensory cells project to motor cells in each of the three neural circuits, we chose to examine the circuit underlying mucus release because $R 2$, the mucus motor cell in the abdominal ganglion, is the earliest cell to be identifiable (Kriegsiein, 1977b). By virtue of its location and size, recordings are possible in metamorphic animals, themselves only about $0.5 \mathrm{~mm}$ in length. Furthermore, mucus is first seen on the body wall at metamorphosis just prior to the onset of juvenile life (Rayport, 1981), so our studies are likely to encompass the full developmental course of mucus release behavior. 


\section{Materials and Methods}

We used juvenile Aplysia (stages 9 to 12) raised from eggs at the Marine Biological Laboratory (Woods Hole, MA) and adults (stage 13) obtained from the wild (Pacific Bio-Marine, Venice, CA). Staging followed criteria developed by Kriegstein (1977) and was further refined by measuring R2 cell diameter, since cell diameter correlates closely with animal age (Ohmori, 1982). Aplysia development (Kriegstein, 1977a) takes approximately 120 days $\left(\right.$ at $22^{\circ} \mathrm{C}$ ) and comprises embryonic, planktonic (stages 1 to 6), metamorphic (stages 7 and 8), and juvenile (stages 9 to 12 ) phases. The adult (stage 13) behaviors emerge during juvenile life (Kriegstein et al., 1974), which extends from about 36 days after hatching to adulthood at 120 days. In the juvenile phase, animals grow from about $0.5 \mathrm{~mm}$ to $8 \mathrm{~mm}$ in body length; R2 soma diameter increases from about $10 \mu \mathrm{m}$ to $200 \mu \mathrm{m}$. Throughout adulthood animals grow, reaching lengths in excess of $300 \mathrm{~mm}$ with R2 soma diameters approaching $1000 \mu \mathrm{m}$.

For physiological recording, juveniles were anesthetized by immersion in a 1:1 mixture of isotonic $\mathrm{MgCl}_{2}$ and sea water for 5 to $10 \mathrm{~min}$. Ganglia were obtained by ultra microdissection after an initial incision was made through the foot. The left connective was cut, and the isolated nervous system was transferred with a glass microprobe to a chamber filled with buffered Aplysia saline. Under low power observation, glass microprobes were placed on the right connective and the siphon and genitopericardial nerves. Suction electrodes were applied to the left connective and branchial nerves. Under high magnification (Fig. 1) with differential interference contrast optics, intracellular recordings were made using micropipettes pulled on a Brown-Flaming Puller (Sutter Instruments, San Francisco, CA) filled with either $3 \mathrm{M} \mathrm{KCl}$ or isotonic $\mathrm{KCl}$, the latter for cells less than $25 \mu \mathrm{m}$ in diameter to avoid osmotic shock. Cell diameters were measured from photographs of the cells taken prior to recording. An explicit guide to microtechnique for juvenile Aplysia exists (Rayport, 1981).

Standard electrophysiological techniques were used. Current was monitored with a virtual ground circuit. Input resistance was monitored throughout the experiment by injection of $0.1-\mathrm{nA}$ hyperpolarizing current pulses using a bridge circuit. Since electrode resistance often changed during penetration of the sheath, the bridge was rebalanced by first adjusting the negative capacitance feedback to square up a calibration pulse, followed by canceling the early transient due to purely resistive characteristics of the electrode so as to align the start of the exponential change in membrane potential with the base line. Recordings were acceptable if cells had action potentials greater than $50 \mathrm{mV}$ in depolarizing amplitude and resting potentials greater than $40 \mathrm{mV}$. In stage 9 animals, recordings from R2 could be made for 5 to $10 \mathrm{~min}$ before the cell began to deteriorate rapidly; this was adequate for this study, which took altogether about $3 \mathrm{~min}$. With stage 10 and onward, stability was not a problem.

To measure homosynaptic depression and heterosynaptic facilitation, R2 was hyperpolarized to $50 \mathrm{mV}$ below resting potential so as to block spiking and to increase PSP size. Stimuli of increasing voltage were delivered to the branchial nerve once every $10 \mathrm{sec}$ until a PSP was evoked. This was taken to be stimulus 1 and the stimulation voltage was thereafter held constant. After stimulus 15, a suprathreshold train was delivered to the left connective. This was judged adequate if it produced at least a $50-\mathrm{mV}$ depolarization or caused $\mathrm{R} 2$ to fire action potentials. Trains which did not meet these criteria produced minimal facilitation.

\section{Results}

In the earliest juvenile animals at stage 9, stable intracellular recordings were obtained from mucus motor cell R2. R2 measured 10 to $15 \mu \mathrm{m}$ in diameter and was twice the size of any other cell in the abdominal ganglion (Fig. 1 shows a view of the right hemiabdominal ganglion focused on R2 in a slightly older stage 10 animal prior to recording). In stage 9 cells, resting potentials measured 40 to $50 \mathrm{mV}$ compared to an adult range of 50 to $60 \mathrm{mV}$. Overshooting action potentials followed by hyperpolarizing afterpotentials similar in shape and time course to adult spikes were seen. Measured input impedances were 200 to 500 megohms, giving membrane resistivities comparable to those seen in the adult. While, undoubtedly, these measurements reflect a degree of cell injury in the smaller cells, the membrane potential, input resistance, and spikes were stable for at least $5 \mathrm{~min}$-long enough to complete the experimental protocol.

Throughout juvenile life (stages 9 to 12) and in adulthood,

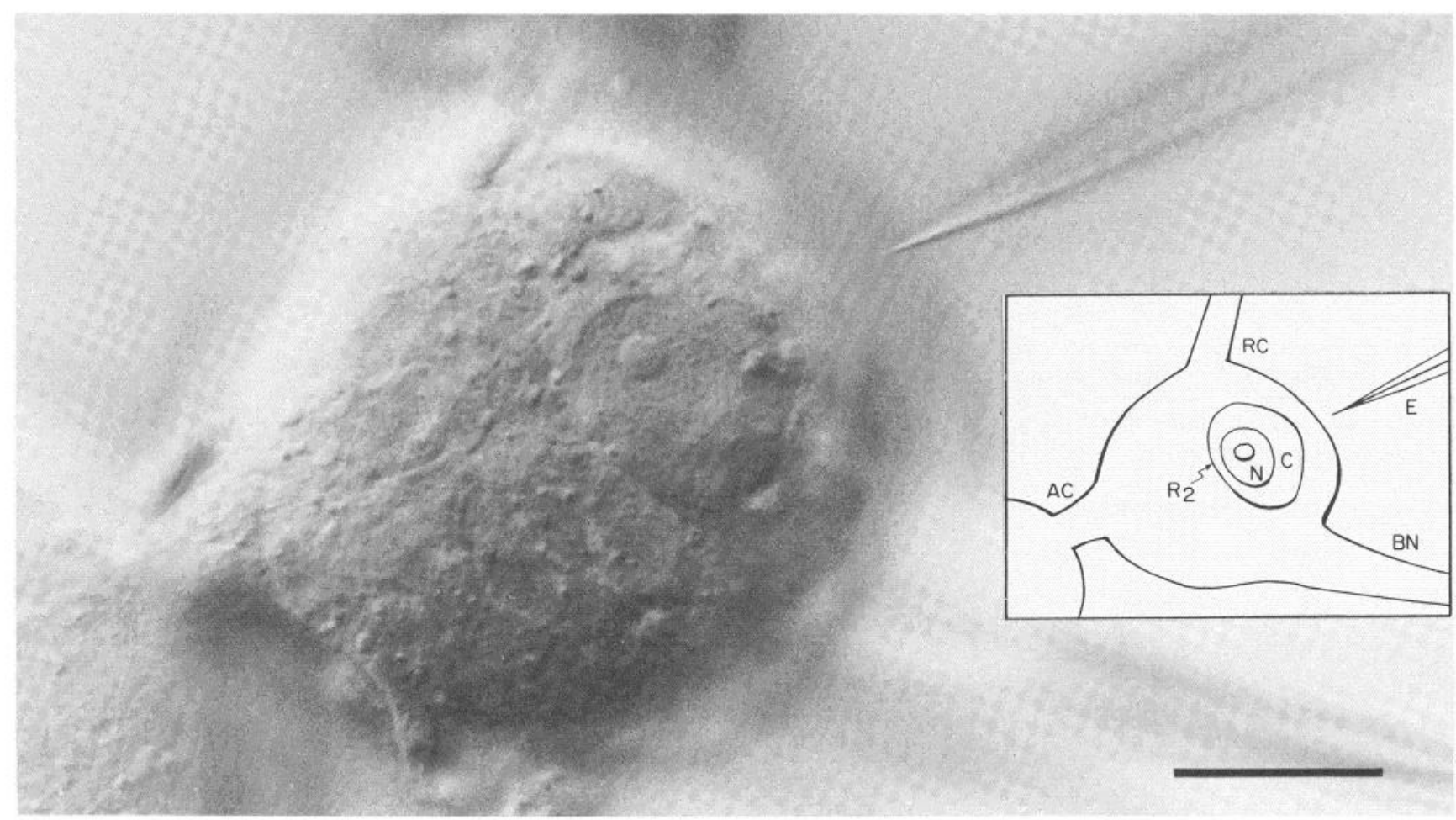

Figure 1. View of the abdominal ganglion under Nomarski optics showing unequivocal identification of R2 (see inset) in stage 10 . The cell measures $26 \mu \mathrm{m}$ in diameter. A well defined nucleus $(N)$, nucleolous, and cytoplasmic $(C)$ structures are seen, as well as an electrode $(E)$ poised for intracellular recording. $R C$, right connective; $B N$, branchial nerve; $A C$, abdominal commissure. Calibration bar $=25 \mu \mathrm{m}$. 
test stimuli to the branchial nerve, as a readout on the sensoryto-motor neuron connection, evoked a complex EPSP in R2. With stimuli once every $10 \mathrm{sec}$, the EPSP progressively decreased in amplitude (Fig. 2). In animals at late stage 9 and early stage 10 , this decrement was indistinguishable from that in the adult (Hawkins et al., 1981) as well as from late stage 10 and stage 11 animals (not illustrated). In each case, an initial rapid decrease was followed by a slower decay, approaching a plateau at about $45 \%$ of the amplitude of the initial EPSP by the fifteenth stimulus. Moreover, just as in the adult, a few minutes of rest between trains of stimuli led to near complete recovery of the response. Therefore, homosynaptic depression is present at the earliest stage studied at the onset of juvenile life.

In contrast, a suprathreshold train to the connective in the same late stage 9 animals had no facilitatory effect on the branchial EPSP (Fig. 3). The connective was clearly intact because this stimulation produced a large barrage of EPSPs in R2. In most cases, EPSP size was reduced after the train to the connective due to the increased membrane conductance resulting from the synaptic input impinging on $\mathrm{R} 2$. This was seen

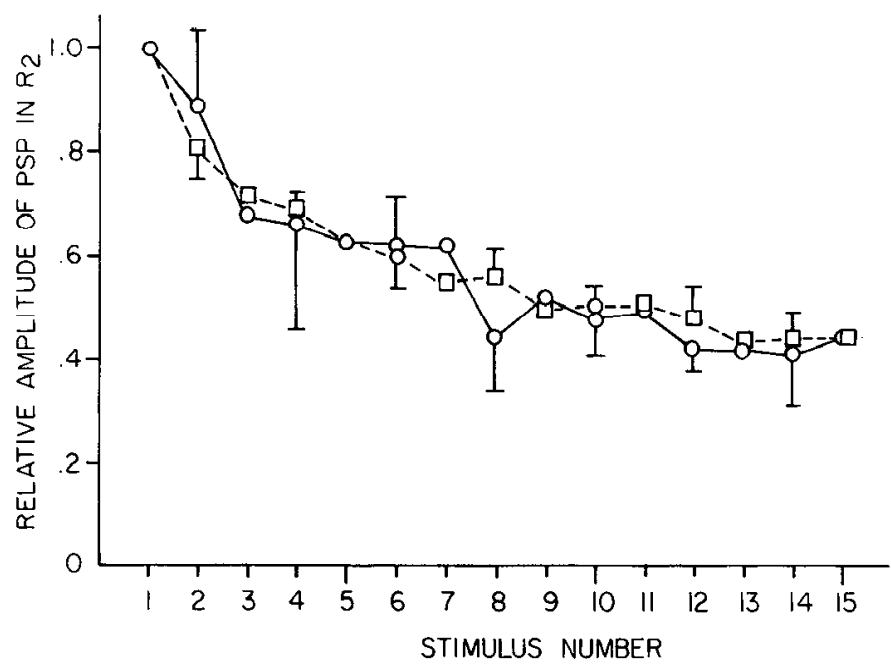

Figure 2. Homosynaptic depression is the same in early postmetamorphic animals and in adults. The relative size of the EPSP in R2 is plotted for each of 15 stimuli to the brachial nerve delivered once every $10 \mathrm{sec}$, normalizing the first EPSP to 1 . Circles represent the average EPSP in animals of stages 9 and $10(N=9)$, and squares represent the EPSPs from adult stage 13 animals $(N=5)$. The bars indicate the standard errors of the mean. In each experiment, the stimulus to the brachial nerve was increased gradually until the appearance of an EPSP in R2; this was then counted as the first stimulus, and in all cases the plot contains data obtained from runs of 15 stimuli in otherwise naive ganglia. There is no significant difference between early juvenile animals and adults. particularly in the juveniles where cell input impedance was 200 to 500 megohms. However, shunting was not sufficient to account for the observed lack of facilitation, as the calculated synaptic current showed no increase and the input impedance of $\mathrm{R} 2$ returned to base line after the first or second stimulus following the connective stimulation; even then no facilitation was evident. In the adult about a 3 -fold increase in the complex EPSP followed the same connective stimulation.

To analyze these data, experiments were grouped in decades of cell diameters, and the experimental results were averaged and compared (Fig. 4A). The amount of facilitation for each decade was quantified by comparing the mean amplitude of the five EPSPs immediately following a tetanus to the five preceding the tetanus (Fig. $4 B$ ). In late stage 9, there was no facilitation; in early stage 10 the amount of facilitation increased gradually, approaching adult levels in stages 11 and 12 .

\section{Discussion}

Our data indicate that a sensory-to-motor neuron synaptic connection in the abdominal ganglion of Aplysia follows a certain developmental sequence in the acquisition of plastic capabilities: homosynaptic depression develops by stage 9 , before heterosynaptic facilitation, and facilitation appears in stage 10 , maturing in stages 11 and 12 . We suggest that the data gleaned from studying the polysynaptic EPSP reflect the development of the monosynaptic connections contained therein, but the small size of the sensory cells as yet prevents direct examination of the monosynaptic connection in younger animals. Behaviorally, habituation is likely to be present prior to sensitization in defensive mucus release. Since LE and RE sensory cells provide afferent input for not only the mucus release circuit but also for the gill withdrawal and inking circuits, and since the sensory neuron terminals are the locus of plasticity, it is likely that the observed developmental sequence for habituation and sensitization may be generalizable to all three defensive behaviors and possibly representative of a still more general tendency.

Parallel studies of another form of plasticity, post-tetanic potentiation (PTP), at two synapses in this ganglion where monosynaptic connections could be examined show that PTP first appears in stage 11 juveniles but does not mature until adulthood, stage 13 (Ohmori et al., 1981; Ohmori, 1982). Of the three forms of plasticity observed during development, heterosynaptic facilitation and PTP, both of which facilitate synaptic transmission, develop days to weeks after the synapses themselves are formed and after depressive capabilities are established at some synapses. Preliminary observations on heterosynaptic (presynaptic) inhibition (J. S. Camardo and S. G. Rayport, unpublished observations), a fourth form of synaptic plasticity, fit this pattern; synapses which show little or no PTP during stage 11 show robust presynaptic inhibition at this

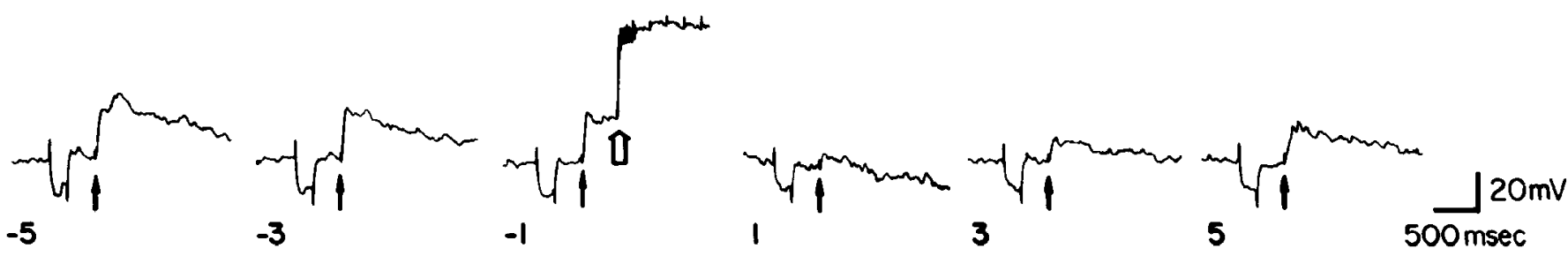

Figure 3. Heterosynaptic facilitation is not present in early postmetamorphic animals. In a late stage 9 animal, stimuli were delivered to the branchial nerve every $10 \mathrm{sec}$ as in the homosynaptic depression experiments (solid arrows); the end of a 15-stimulus run is shown. Each stimulus was preceded by a $0.1-\mathrm{nA}, 200-\mathrm{msec}$ intracellular current pulse to measure the input impedance of R2. A train of suprathreshold stimuli at $5 / \mathrm{sec}$ for $5 \mathrm{sec}$ was delivered to the left connective (open arrow) interposed between the 15 th and 16th branchial nerve stimulations. A small number of axonal spikes are seen after the first stimulus of the train. No increase in the size of the EPSP in R2 following connective stimulation was seen, up to and beyond the fifth postconnective stimulus to the branchial nerve. Typically, about a $30 \%$ decrease in input impedance was seen during the first two to three post-train stimuli, but this is insufficient to account for the lack of facilitation. In the adult, in striking contrast, facilitation of the EPSP occurs within one or two stimuli following the left connective train and lasts considerably longer (Hawkins et al., 1981). 

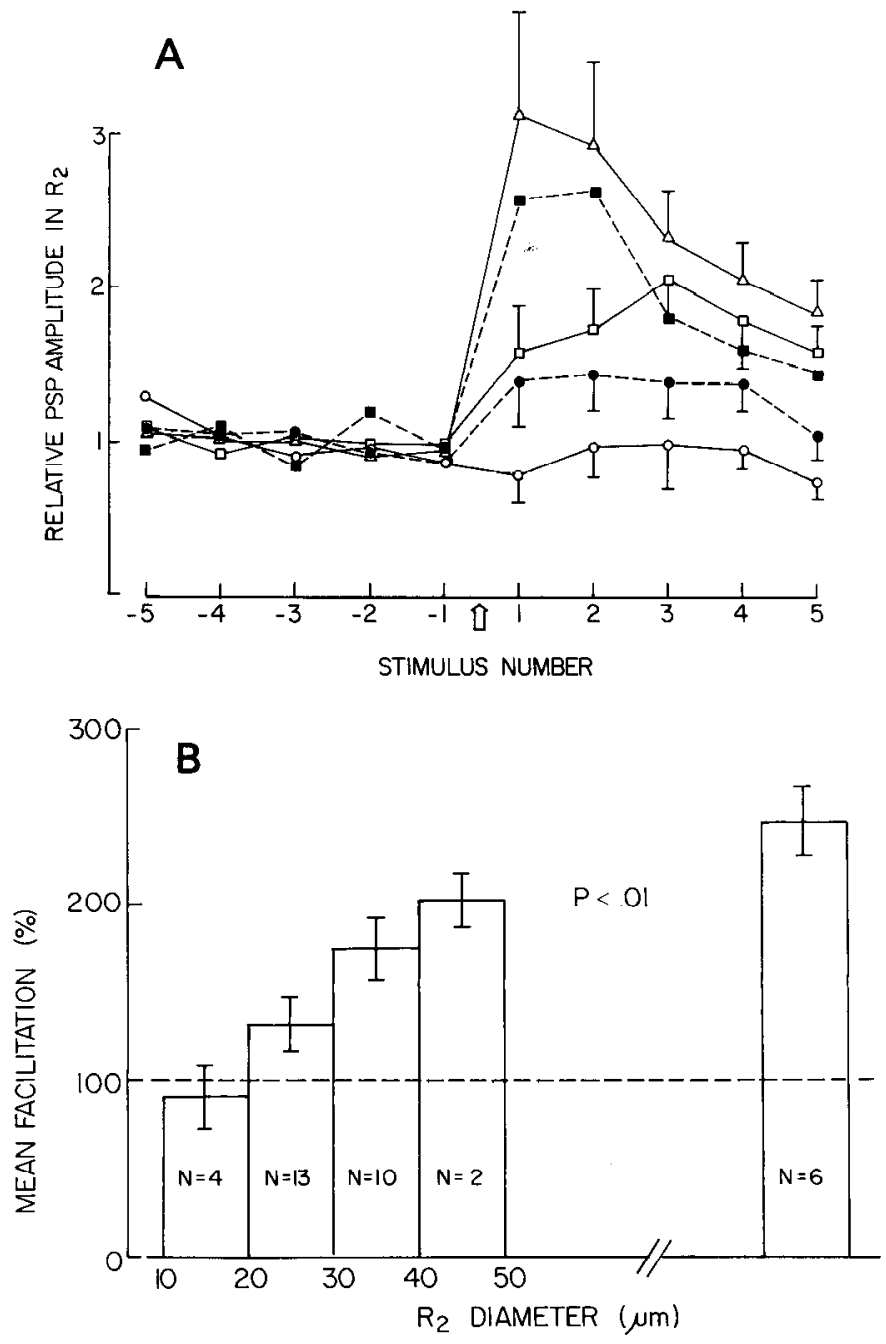

STAGE

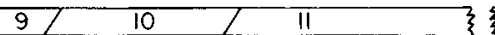

13

Figure 4. Development of heterosynaptic facilitation. $A$, Normalized EPSP amplitude of the five stimuli before and after connective stimulation in juvenile and adult animals is shown. The experiments were divided into bins of $\mathrm{R} 2$ diameter: open circles represent $\mathrm{R} 2$ diameters of 10 to $20 \mu \mathrm{m}(N=4)$, solid circles of 20 to $30 \mu \mathrm{m}(N=13)$, open squares of 30 to $40 \mu \mathrm{m}(N=10)$, solid squares of 40 to $50 \mu \mathrm{m}(N=2)$, and triangles of the adult $(N=6)$, in which $\mathrm{R} 2$ is 500 to $1000 \mu \mathrm{m}$ in diameter. The last five stimuli of the homosynaptic depression experiment are shown with the mean of the five stimuli normalized to 1 . The train of stimuli to the left connective is marked by the open arrow and produced increasing amounts of facilitation with maturation. $B$, To summarize, the mean facilitation by cell diameters is shown. The five EPSPs preceding the left connective train and the five following are each averaged. The amount of facilitation is the postconnective average divided by the preconnective average; a mean facilitation of $100 \%$, therefore, represents no facilitation. The EPSPs were averaged for each animal individually, and the bars represent the standard error of the percentage of facilitation for all of the animals of each group averaged together. There is a gradual increase in the mean facilitation with maturation; the adult level of facilitation is achieved during stages 11 and 12. The relationship between developmental stage and the diameter of R2 is shown at the bottom of the figure. The trend is significant at the 0.01 level using a one-way analysis of variance.

same stage. ${ }^{3}$ This suggests that mechanisms which depress synaptic transmission develop before mechanisms which facilitate transmission.

We suggest that the development of synaptic plasticity en-

${ }^{3}$ In early stage 11 animals we have observed that the monosynaptic potential elicited in cell R15 by stimulation of the right connective tails specific changes in the properties of synapses and that these changes occur in a defined sequence after the establishment of effective synaptic connections. These changes may entail the growth of modulatory heterosynaptic connections or maturation of the presynaptic cells or both. The earlier appearance of depressive synaptic plasticities might serve to maintain synaptic connections in a minimally effective state, preventing inappropriate neuronal activity from having adverse behavioral consequences in advance of the development of necessary higher order neuronal circuitry. The development of facilitation would then serve functionally to activate synapses by increasing their efficacy and to define the time when the mediated hehavior would be susceptible to environmental influence. Perturbations at such critical periods might profoundly affect the properties of maturing synapses, perhaps permanently modifying synaptic properties and the capacity for learning in the adult. Elucidating the mechanisms underlying development of synaptic plasticity should offer important developmental insights and may allow dissection of subcellular mechanisms of synaptic plasticity.

Note added in proof. Rankin and Carew (personal communication) in recent behavioral experiments have examined siphon withdrawal starting with late stage 9 juveniles. At this stage, preliminary observations suggest that habituation is present as soon as the siphon has developed sufficiently to permit measurements. Only later in stage 10 does sensitization begin to appear.

\section{References}

Byrne, J. H. (1980) Neural circuit for inking behavior in Aplysia. J. Neurophysiol. 43: 896.

Carew, T. J., and E. R. Kandel (1977) Inking in Aplysia californica. I. Neural circuit of an all-or-none behavioral response. J. Neurophysiol. 40: 692 .

Castellucci, V. F., and E. R. Kandel (1974) A quantal analysis of the synaptic depression underlying habituation of the gill-withdrawal reflex in Aplysia. Proc. Natl. Acad. Sci. U. S. A. 71: 5004.

Castellucci, V. F., and E. R. Kandel (1976) Presynaptic facilitation as a mechanism for behavioral sensitization. Science 194: 1176.

Castellucci, V. F., H. Pinsker, I. Kupfermann, and E. R. Kandel (1970) Neuronal mechanisms of habituation and dishabituation of the gillwithdrawl reflex in Aplysia. Science 167: 1745.

Hawkins, R. D., V. F. Castellucci, and E. R. Kandel (1981) Interneurons involved in mediation and modulation of the gill-withdrawal reflex in Aplysia. II. Identified neurons produce heterosynaptic facilitation contributing to behavioral sensitization. J. Neurophysiol. 45: 315.

Hebb, D. O. (1949) The Organization of behavior: A Neuropsychological Theory, John Wiley \& Sons, Inc., New York.

Kandel, E. R. (1977) Cellular Insights into Behavior and Learning, The Harvey Lectures, Series 73, Academic Press, Inc., New York.

Kandel, E. R., and J. H. Schwartz (1982) Molecular biology of learning: Modulation of transmitter release. Science 218: 433.

Klein, M., E. Shapiro, and E. R. Kandel (1980) Synaptic plasticity and the modulation of the $\mathrm{Ca}^{++}$current. J. Exp. Biol. 89: 117.

Konorski, J. (1948) Conditioned Reflexes and Neuron Organization, Cambridge University Press, Cambridge, England.

Kriegstein, A. R. (1977a) Stages in the post-hatching development of Aplysia californica. J. Zool. 199: 275.

Kriegstein, A. R. (1977b) Development of the nervous system of Aplysia californica. Proc. Natl. Acad. Sci. U. S. A. 74: 375.

Kriegstein, A. R., V. Castellucci, and E. R. Kandel (1974) Metamor-

$\left(\mathrm{RC}_{1}-\mathrm{R}_{15}\right)$ undergoes very little post-tetanic potentiation, while a strong decrease in the size of the EPSP occurs with stimulation of the left connective, and this decrease is not different from that seen at the same synapse in the adult (J. S. Camardo and S. G. Rayport, unpublished observations). Furthermore, the same pattern appears to hold for synapses developing in culture between L10 and left upper quadrant cells which in the adult show presynaptic inhibition and post-tetanic potentiation (S. Schacher and J. S. Camardo, unpublished observations). 
phosis of Aplysia californica in laboratory culture. Proc. Natl. $\Lambda$ cad. Sci. U. S. A. 71: 3654.

Kupfermann, I., V. F. Castellucci, and H. Pinsker (1970) Neuronal correlates of habituation and dishabituation of the gill-withdrawal reflex in Aplysia. Science 167: 1743.

Kupfermann, I., T. J. Carew, and E. R. Kandel (1974) Local, reflex and central commands controlling gill and siphon movements in Aplysia. J. Neurophysiol. 37: 996.

Ohmori, H. (1982) Development of post-tetanic potentiation at identified inhibitory and excitatory synapses in Aplysia. J. Physiol.
(Lond.) 322: 223.

Ohmori, H., S. G. Rayport, and E. R. Kandel (1981) Emergence of post-tetanic potentiation as a distinct phase in the differentiation of an identified synapse in Aplysia. Science 213: 1016.

Rayport, S. G. (1981) Development of the functional and plastic capabilities of neurons mediating a defensive behavior in aplysia. Doctoral dissertation, Columbia University, New York.

Rayport, S. G., R. T. Ambron, and J. Babiarz (1983) Identified cholinergic neurons $\mathrm{R} 2$ and $\mathrm{LPl}_{1}$ control mucus release in Aplysia. J. Neurophysiol. 49: 864 . 